\title{
Template Synthesized Molecularly Imprinted Polymer Nanotube Membranes for Chemical Separations
}

\author{
Hai-Juan Wang, Wen-Hui Zhou, Xiao-Fei Yin, Zhi-Xia Zhuang, Huang-Hao Yang* and \\ Xiao-Ru Wang* \\ Contribution from Qingdao Key Lab of Analytical Technology Development, The First \\ Institute of Oceanography, SOA, Qingdao, 266061, P.R. China
}

\section{Supporting Information}

\section{Materials.}

A commercially available anodic alumina oxide membrane with a thickness of $60 \mu \mathrm{m}$ and a quoted pore diameter of $100 \mathrm{~nm}$ was purchased from Whatman. 3aminopropyltrimethoxysilane (APTMOS), 4-vinylpyridine (4-VP), ethylene glycol dimethacrylate (EGDMA), $\mathrm{CuBr}$ and 1,4,8,11-tetraazacyclotetradecane (Me4Cyclam) were purchased from Aldrich. Phenol, $\beta$-estradiol, estrone and cholesterol were purchased from Sigma.

\section{Modifying AAO membrane with 3-aminopropyltrimethoxysilane}

This was accomplished by immersing the AAO membrane into a solution prepared by mixing $0.5 \mathrm{~mL}$ of 3-aminopropyltrimethoxysilane with $3 \mathrm{~mL}$ ethanol that containing 0.2 $\mathrm{mL}$ of sodium acetate buffer solution $(50 \mathrm{mM}, \mathrm{pH}=5.0)$. The flask containing the above solution was vacuumized for $5 \mathrm{~min}$ to remove air from the pores of the membrane. The membrane remained in this solution for another 15 min under ambient pressure before being rinsed with ethanol. The membrane was then cured by heating in vacuum at 150 ${ }^{\circ} \mathrm{C}$ for $1 \mathrm{~h}$. Note that the surfaces of the membrane are coated with thin silica film, which are removed by a brief mechanical polish.

\section{Immobilizing of ATRP initiator}


The ATRP initiator, 2-Bromo-2-methylpropionyl bromide, was then grafted onto the silanized AAO membrane by dipping the membrane in $4 \mathrm{~mL}$ of dry dichloromethane containing dry triethylamine $(4 \%, \mathrm{v} / \mathrm{v})$. Afterward, $4 \mathrm{~mL}$ of dry dichloromethane containing $0.016 \mathrm{~mL}$ of 2-Bromo-2-methylpropionyl bromide was slowly dropped into the solvent. The mixture was kept first at $0{ }^{\circ} \mathrm{C}$ for 2 hours and then at room temperature for 12 hours. The AAO membrane was repeatedly rinsed with acetone and toluene before being dried under vacuum.

\section{Scatchard analysis}

The amount of $\beta$-estradiol bound to the molecularly imprinted nanotubes, expressed as $\boldsymbol{B}$, was plotted versus varied initial $\beta$-estradiol concentration and a typical Scatchard plot for $\beta$-estradiol was shown in Figure S1. Two straight lines fitting the Scatchard equation, $\boldsymbol{B} / \boldsymbol{F}=\left(\boldsymbol{B}_{\max }-\boldsymbol{B}\right) / \boldsymbol{K}_{\mathrm{d}}$, can be drawn.

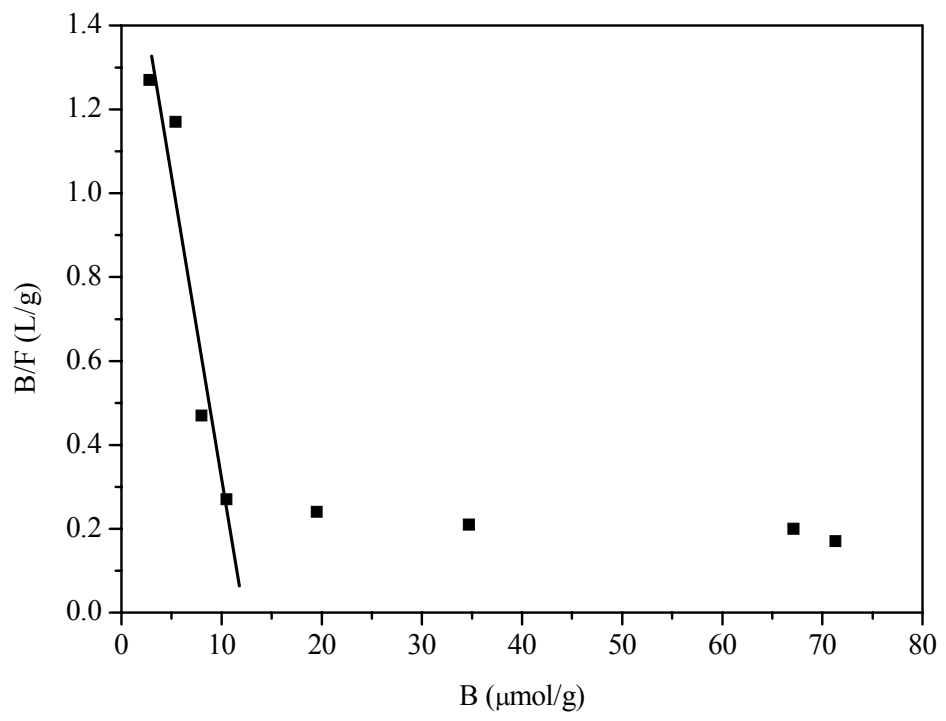

Figure S1 Scatchard plot for $\beta$-estradiol binding to the molecularly imprinted nanotubes.

$\boldsymbol{F}$ was the concentration of free $\beta$-estradiol. The dissociation constant, $\boldsymbol{K}_{\mathrm{d}}$ and the maximum number of binding site, $\boldsymbol{B}_{\max }$, were estimated to be $7.25 \mu_{\mathrm{mol}} / \mathrm{L}$ and $12.38 \mu \mathrm{mol} / \mathrm{g}$. 


\section{Wall thickness of the MIP nanotubes}

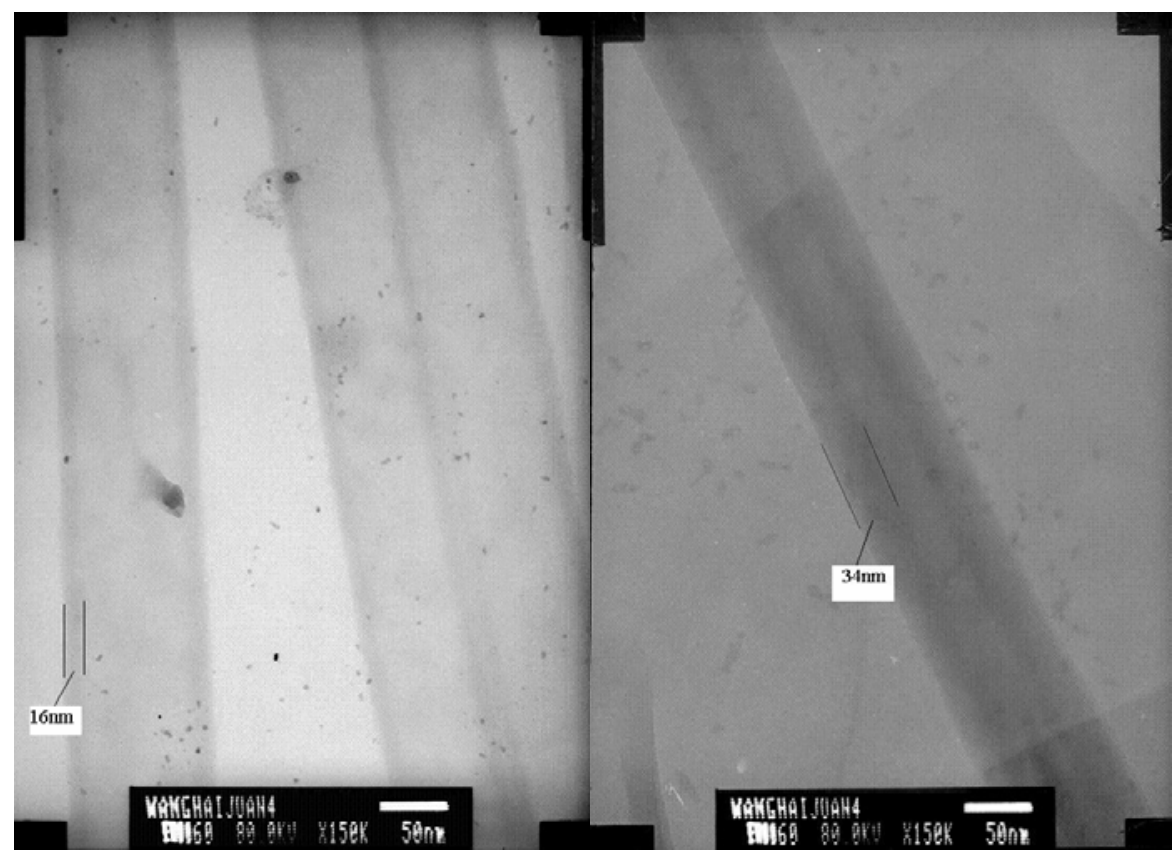

Figure S2 TEM of the molecularly imprinted nanotubes. (Left) polymerization time was $24 \mathrm{~h}$. (Right) polymerization time was $48 \mathrm{~h}$ 